\title{
CORRELAÇÃO DA QUALIDADE VIDA E SONOLÊNCIA DIURNA EM ESTUDANTES DE FISIOTERAPIA
}

\section{CORRELATION OF QUALITY LIFE AND DAYTIME SLEEPINESS IN PHYSIOTHERAPY STUDENTS}

George Alberto da Silva Dias (ORCID: 0000-0002-9807-6518) ${ }^{1}$

Aline Silva Castro (ORCID: 0000-0002-1554-3126)

Leonardo Breno do Nascimento de Avizl ORCID: 0000-0003-1760-660X) ${ }^{1}$

Shaumin Vasconcelos Wu (ORCID: 0000-0002-1954-6068)

Jamylle Silva Campos (ORCID: 0000-0003-1909-8071) ${ }^{1}$

Angélica Homobono Nobre (ORCID: 0000-0001-8067-4993) ${ }^{1}$

Biatriz Araújo Cardoso Dias (ORCID: 0000-0002-4985-2779)

\begin{abstract}
RESUMO
Distúrbios do ciclo sono/vigília são considerados um problema de saúde pública e repercutem diretamente na qualidade de vida. Assim, objetivou-se correlacionar a qualidade de vida e a sonolência diurna em estudantes de fisioterapia. Trata-se de um estudo observacional, descritivo e analítico do tipo transversal. Participaram do estudo 123 discentes do curso de Fisioterapia, sendo selecionados por meio da amostragem não probabilística por conveniência. Para a análise da qualidade de vida, foi utilizado o questionário World Health Organization Quality of Life Assessment (WHOQOL-bref), e para sonolência diurna, a escala de sonolência de Epworth. Utilizaram-se os testes análise de variância (Anova um critério) com post hoc de Teste $t$, o teste $t$ Student e a regressão linear múltipla, com nível alfa de significância de $5 \%(\mathrm{p} \leq 0,05)$. Observou-se que o quarto ano do curso apresentou pior resultado para qualidade de vida no domínio físico e ambiente, e ao se comparar com as outras séries do curso, apenas o domínio físico foi menor. Com relação à sonolência diurna, não foi observada diferença significante entre os anos. Porém, ao realizar a correlação da qualidade de vida com a sonolência diurna, houve correlação para os alunos do primeiro e segundo. Assim, conclui-se que, para qualidade de vida, o ano mais acometido é o quarto, principalmente no domínio físico. Ademais, não houve diferença com relação à sonolência. No entanto, quando os alunos do primeiro ano apresentam melhor percepção da qualidade de vida no domínio relação social, mais sonolentos eles se percebem; e quanto melhor a percepção no domínio ambiente, menos sonolentos eles se percebem. Outrossim, nos alunos do segundo ano, quanto melhor percepção da qualidade de vida no domínio relações sociais, menos sonolentos eles estão.
\end{abstract}

Palavras-chave: Qualidade de vida; Sonolência; Fisioterapia
Autor correspondente: George Alberto da Silva Dias E-mail: george@uepa.br

${ }^{1}$ Departamento de Ciência do Movimento Humano, Universidade do Estado do Pará.

\begin{abstract}
Sleep/wake cycle disorders are considered a public health problem and have a direct impact on quality of life. Thus, our aim was to correlate quality of life and daytime sleepiness in physiotherapy students. This is an observational, descriptive and analytical cross-sectional study. 123 students of the Physiotherapy course participated in the study, being selected through non-probabilistic sampling for convenience. The World Health Organization Quality of Life Assessment (WHOQOL-bref) questionnaire was used to analyze quality of life and the Epworth sleepiness scale for daytime sleepiness. Analysis of variance tests (Anova one criterion) with post hoc $t$ test, Student $t$ test and multiple linear regression were used, with an alpha level of significance of $5 \%(p \leq 0.05)$. It was observed that the fourth year of the course had a worse result for quality of life in the physical and environmental domains and when comparing with the other series of the course, only the physical domain was smaller. Regarding daytime sleepiness, there was no significant difference between the years. However, when correlating quality of life with daytime sleepiness, there was a correlation for students in the first and second. Thus, it is concluded that for quality of life, the year most affected is the fourth year mainly in the physical domain. There was also no difference regarding sleepiness. However, when first year students have a better perception of quality of life in the social relationship domain, the sleepier they perceive themselves, and the better the perception in the environmental domain, the less sleepy they perceive themselves. In addition, with the second year students, the better perception of quality of life in the social relations domain, the less sleepy they are.
\end{abstract}




\section{INTRODUÇÃO}

Qualidade de Vida (QV), de acordo com a Organização Mundial da Saúde (OMS), é a percepção do indivíduo sobre a sua posição na vida, no contexto da cultura e dos sistemas de valores nos quais ele vive, e em relação a seus objetivos, expectativas, padrões e preocupações. Ela torna-se relevante e precisa ser avaliada em múltiplas facetas, principalmente quando se trata de acadêmicos da área da saúde ${ }^{1}$.

A universidade proporciona diversas vivências que podem repercutir positiva ou negativamente na QV do discente. Nesse período de inserção no ensino superior, culmina-se com uma fase delicada da vida do ser humano, que é a transição da adolescência para a vida adulta. $\mathrm{O}$ indivíduo passa a experimentar modificações e adaptações inerentes a esse novo ciclo vital juntamente com mudanças acadêmicas mediante o ingresso na universidade ${ }^{2}$.

Hoje em dia, a saúde mental dos universitários ganhou destaque, em que o curso de Fisioterapia não foge à regra, pois ele impõe estresse e tensões nos discentes. Há relatos que as atividades acadêmicas causam desgaste físico e emocional, impactando diretamente em sua QV1. Assim, as atividades extracurriculares, a relação professor-aluno, a convivência entre os universitários, bem como a evolução do curso até a sua formatura, podem ser fatores que alterem a percepção da QV por parte dos estudantes. Aliada a isso, a carga horária excessiva pode impossibilitar atividades de lazer, sendo que estas poderiam minimizar esses transtornos ${ }^{3,4}$.

Todas essas experiências podem provocar alterações na QV dos acadêmicos, como também promover alterações do sono, culminando para o sedentarismo, isolamento social, dificuldade de concentração, ansiedade, irritabilidade e depressão, afetando diretamente a saúde mental dos alunos ${ }^{5}$.
O sono é um componente importante para a sobrevivência, possuindo várias funções, como liberação hormonal e processamento de memória. Outrossim, isso pode ser influenciado por fatores sociais, incluindo rotinas familiares e da própria universidade ${ }^{6}$.

Vários dilemas enfrentados por eles, como deixar o convívio familiar para estudar na universidade e a inserção em um novo ambiente escolar, podem ser fatores fortes o suficiente para levar a alterações no ciclo sono vigília. Decidir entre manter uma regularidade de sono e um bom rendimento escolar é uma situação desgastante que pode influenciar em sua $\mathrm{QV}^{6,7}$.

Distúrbios do ciclo sono vigília são considerados um problema de saúde pública e repercutem diretamente na QV. Perturbações no ciclo acarretam diminuição do estado de alerta e concentração, consequentemente, alteram o desenvolvimento de atividades acadêmicas? ${ }^{7}$.

A importância e a relevância de serviços de orientação ao aluno, para atender esse indivíduo de forma preventiva e assistencial, são fundamentais para que se possa evitar um agravamento dos sintomas psicológicos, além de diminuir os efeitos negativos aos aspectos pessoais e sociais da vida do aluno. Com isso, este estudo objetiva correlacionar a QV e a sonolência diurna em estudantes de diferentes períodos do curso de Fisioterapia pertencentes ao ensino por metodologia ativa e tradicional.

\section{MÉTODOS}

\section{Amostra}

Participaram do estudo 123 discentes do curso de Fisioterapia da Universidade do Estado do Pará (Uepa) 
regularmente matriculados, sendo selecionados por meio da amostragem não probabilística por conveniência, avaliados no período de agosto a dezembro de 2018. Desse total, 72 alunos pertenciam ao ensino por metodologia ativa (Aprendizagem Baseada em Problemas - ABP); e 51 alunos, à Metodologia Tradicional (TD).

Como critérios de inclusão, optou-se por discentes de ambos os sexos, maiores e menores de 18 anos do curso de Fisioterapia da Uepa e que quisessem participar da pesquisa. Foram excluídos aqueles que não assinaram formalmente a sua participação no estudo e que se recusaram a responder a um dos questionários propostos.

\section{Procedimentos e instrumentos de coleta de dados}

Os instrumentos de coleta de dados utilizados na pesquisa foram autoaplicáveis, facilitando a fidedignidade nas respostas. No primeiro momento, os participantes encontravam-se dentro do ambiente universitário no qual foram abordados e explicado o estudo. No segundo momento, após o consentimento dos participantes, distribuíram-se os questionários aos alunos para o autopreenchimento das informações, podendo ser entregue no dia seguinte após seu consentimento.

Para a QV, o questionário World Health Organization Quality of Life Assessment (WHOQOL-bref) foi o instrumento de pesquisa utilizado. Este é constituído de 26 perguntas (as duas primeiras perguntas se referem à QV geral). Esse instrumento é uma versão abreviada do questionário WHOQOL-100 que contém 100 perguntas. As respostas seguem a escala de Likert, que compreende a pontuações de 1 a 5: quanto maior a pontuação, melhor a QV. Fora essas duas questões (1 e 2), o instrumento tem 24 facetas que compõem 4 domínios, que são: físico, psicológico, relações sociais e meio ambiente ${ }^{8}$.

Para a avaliação da sonolência, utilizou-se a escala de sonolência de Epworth. Esta avalia a probabilidade de adormecer, contendo oito situações que envolvem atividades de vida diária simples, questionando sobre a possibilidade de o indivíduo cochilar em cada circunstância. As respostas seguem a escala de Likert, que compreende a pontuações de 0 (nenhuma chance de cochilar) e 3 (alta chance de cochilar). A pontuação total varia de 0 a 24 , sendo que os resultados entre 0 e 10 indicam ausência de sonolência; entre 10 e 16, sonolência leve; entre 16 e 20, sonolência moderada; e entre 20 e 24 pontos, sonolência excessiva 9 .

\section{Análise estatística}

Adotou-se o software Excel ${ }^{\circledR}$ 2010 para entrada dos dados e confecção das tabelas, bem como o BioEstat 5.0 na análise estatística. As variáveis categóricas foram apresentadas como frequências; e as numéricas, por meio de medidas de tendência central e dispersão. Utilizaram-se os testes análise de variância (Anova um critério) com post hoc de Teste $t$, $o$ teste $t$ Student e a regressão linear múltipla. Adotou-se o nível alfa de significância de 5\% $(\mathrm{p} \leq 0,05)$.

\section{Aspectos éticos}

A pesquisa teve início após aprovação do Comitê de Ética em Pesquisa da Uepa (Parecer $n^{\circ}$ 2.710.595). Trata-se de um estudo observacional, descritivo e analítico, do tipo transversal, seguindo as normas da Resolução n 466/12 do Conselho Nacional de Saúde relativa à pesquisa com seres humanos. Todos os participantes assinaram o Termo de Consentimento Livre e Esclarecido e/ou o Termo de Assentimento Livre e Esclarecido.

\section{RESULTADOS}

Dos 123 discentes, 33 alunos foram do primeiro ano (idade: 19,27 $\pm 1,84$ anos), 24 do segundo (Idade: 19,41 $\pm 1,66$ anos), 15 do terceiro (Idade: 19,87 $\pm 0,61$ anos), 26 do quarto (Idade: $22,69 \pm 2,46$ 


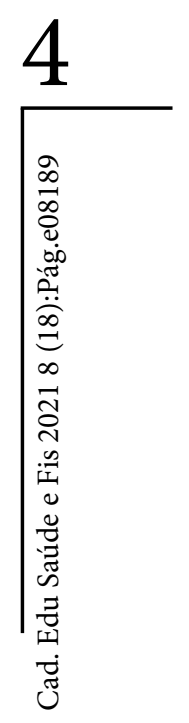

anos) e 25 do quinto ano (Idade: $22,60 \pm$ 1,50 anos). Em grande maioria, os alunos são de etnia parda, solteiros e com renda familiar de 2 a 6 salários, conforme mostra a tabela 1

Tabela 1. Perfil sociodemográfico dos alunos do curso de Fisioterapia da Uepa, Belém-Pará, 2018

\begin{tabular}{|c|c|c|c|c|c|}
\hline \multirow{2}{*}{ Variáveis } & \multicolumn{3}{|c|}{ ABP } & \multicolumn{2}{|c|}{ TD } \\
\hline & $1^{\circ}$ ano & $2^{\circ}$ ano & $3^{\circ}$ ano & $4^{\circ}$ ano & $5^{\circ}$ ano \\
\hline \multicolumn{6}{|l|}{ Sexo } \\
\hline Masculino & $11(33,3 \%)$ & $5(20,8 \%)$ & $7(46,7 \%)$ & $7(26,9 \%)$ & $5(20,0 \%)$ \\
\hline Feminino & $22(66,7 \%)$ & $19(79,2 \%)$ & $8(53,3 \%)$ & $19(73,1 \%)$ & $20(80,0 \%)$ \\
\hline \multicolumn{6}{|l|}{ Etnia } \\
\hline Branca & $5(15,2 \%)$ & $2(8,3 \%)$ & $4(25,0 \%)$ & $4(15,4 \%)$ & $6(24,0 \%)$ \\
\hline Parda & $9(27,3 \%)$ & $14(58,3 \%)$ & $9(56,3 \%)$ & $13(50,0 \%)$ & $11(44,0 \%)$ \\
\hline Negra & $3(9,1 \%)$ & $4(16,7 \%)$ & $2(12,5 \%)$ & $1(3,8 \%)$ & $5(20,0 \%)$ \\
\hline Não declarou & $16(48,5 \%)$ & $4(16,7 \%)$ & $1(6,2 \%)$ & $8(30,8 \%)$ & $6(24,0 \%)$ \\
\hline \multicolumn{6}{|l|}{ Estado civil } \\
\hline Solteiro & $32(97,0 \%)$ & $24(100,0 \%)$ & $16(100,0 \%)$ & $24(92,3 \%)$ & $25(100,0 \%)$ \\
\hline Casado & $1(3,0 \%)$ & - & - & $2(7,7 \%)$ & - \\
\hline \multicolumn{6}{|l|}{ Renda familiar } \\
\hline Não declarou & $1(3,0 \%)$ & - & - & - & - \\
\hline$<1$ salário & $2(6,1 \%)$ & - & $1(6,7 \%)$ & $1(3,8 \%)$ & - \\
\hline 1 salário & $3(9,1 \%)$ & $4(16,7 \%)$ & $1(6,7 \%)$ & $2(7,7 \%)$ & $2(8,0 \%)$ \\
\hline 2 a 3 salários & $14(42,4 \%)$ & $12(50,0 \%)$ & $5(33,3 \%)$ & $15(57,7 \%)$ & $10(40,0 \%)$ \\
\hline 5 a 6 salários & $9(27,3 \%)$ & $6(25,0 \%)$ & $6(40,0 \%)$ & $5(19,2 \%)$ & $7(28,0 \%)$ \\
\hline$>7$ salários & $3(9,1 \%)$ & $2(8,3 \%)$ & $2(13,3 \%)$ & $3(11,5 \%)$ & $6(24,0 \%)$ \\
\hline
\end{tabular}

ABP - Aprendizagem Baseada em Problemas; TD - Metodologia Tradicional

(-) Dados numéricos igual a zero. 
Ao se analisar a QV separadamente por período do curso, foi possível notar resultados interessantes. Apenas o quarto ano apresentou resultados significantes ( $p=0,04)$, em que foi observado que houve pior percepção no domínio físico quando comparado a relações sociais ( $p=0,02)$, e pior percepção no domínio ambiente quando comparado a relações sociais $(p=0,007)$. Ou seja, os domínios físico e ambiente foram os que tiveram pior percepção.
Quando se compararam os alunos por período do curso, houve resultado significante $(p=0,001)$ apenas para o domínio físico, em que os alunos do primeiro, segundo, terceiro e quinto ano apresentaram melhor percepção quando comparados aos alunos do quarto ano. Quando se analisam os alunos estratificados pelo tipo de metodologia em que estão inseridos, nota-se que, entre os alunos da metodologia ativa, a percepção sobre a QV é semelhante; o que não se pode dizer em relação à $\mathrm{TD}$, pois foi observado que os alunos do quarto ano apresentam pior percepção quando comparados aos alunos do quinto ano $(\mathrm{p}<0,001)$. Para os demais domínios, a percepção foi semelhante para ambas as metodologias, conforme mostra a tabela 2 .

Tabela 2. Comparação da qualidade de vida entre as metodologias de aprendizagem e os alunos do curso de Fisioterapia da Uepa, Belém-Pará, 2018

\begin{tabular}{|c|c|c|c|c|c|c|}
\hline \multirow{2}{*}{ Domínios } & \multicolumn{3}{|c|}{ ABP } & \multicolumn{2}{|c|}{ TD } & \multirow{2}{*}{ p-valor ${ }^{2}$} \\
\hline & $1^{\circ}$ ano & $2^{\circ}$ ano & $3^{\circ}$ ano & $4^{\circ}$ ano & $5^{\circ}$ ano & \\
\hline Físico & 3,41 & 3,43 & 3,32 & 2,93 & 3,58 & ${ }^{* *} 0,001$ \\
\hline Psicolóaico & 3,25 & 3,22 & 3,38 & 3,16 & 3,38 & 0,57 \\
\hline Relações sociais & 3,49 & 3,55 & 3,63 & 3,39 & 3,90 & 0,10 \\
\hline Ambiente & 3,06 & 3,26 & 3,04 & 2,86 & 3,29 & 0,55 \\
\hline Autoavaliação da Qualidade de Vida & 3,24 & 3,54 & 3,37 & 3,23 & 3,45 & 0,72 \\
\hline p-valor ${ }^{1}$ & 0,16 & 0,66 & 0,26 & $0,04^{\star}$ & 0,052 & \\
\hline
\end{tabular}

ABP - Aprendizagem Baseada em Problemas; TD - Metodologia Tradicional. (p-valor1) Comparação intragrupo; (p-valor2) comparação intergrupo.

* Resultado estatisticamente significante; teste Anova, $\mathrm{p} \leq 0,05$. Comparações significantes para oA $4^{\circ}$ ano: físico $x$ relações sociais $(p=0,02)$; relações sociais $x$ ambiente $(p=0,007)$.

** Resultado estatisticamente significante; teste Anova, $p \leq 0,05$. Comparações significantes no domínio Físico: $1^{\circ} \times 4^{\circ}$ ano $(p<0,001) ; 2^{\circ} \times 4^{\circ}$ ano $(p<0,002) ; 3^{\circ} \times 4^{\circ}$ ano $(p<0,03) ; 4^{\circ} \times 5^{\circ}$ ano $(p<0,001)$. 
Com relação à sonolência, em uma primeira análise, foi possível observar que a maioria dos alunos do primeiro ao terceiro ano apresentou sonolência leve (48,5\%; 45,8\% e 43,8\% respectivamente); já quanto aos alunos do quarto e quinto ano, a maioria tinha ausência de sonolência ( $50 \%$ e 52\% respectivamente). Porém, ao classificar entre aqueles que tinham ou não sonolência, foi possível notar que 54,6\% dos alunos do primeiro apresentaram sonolência, $58,3 \%$ no segundo ano, $56,4 \%$ no terceiro ano, 49,9\% para o quarto ano e $48 \%$ para o quinto ano, conforme mostra a tabela 3. Apesar de não mostrar resultados significativos, pode-se inferir que são valores altos para todos os anos do curso.

Tabela 3. Análise descritiva da sonolência dos alunos do curso de Fisioterapia da Uepa, Belém-Pará, 2018

\begin{tabular}{lccccc}
\hline \multirow{2}{*}{ Escala de sonolência } & \multicolumn{3}{c}{ ABP } & \multicolumn{2}{c}{ TD } \\
\cline { 2 - 4 } \cline { 5 - 6 } & $1^{\circ}$ ano & $2^{\circ}$ ano & $3^{\circ}$ ano & $4^{\circ}$ ano & $5^{\circ}$ ano \\
\hline Ausência de sonolência & $15(45,5 \%)$ & $10(41,7 \%)$ & $7(43,8 \%)$ & $13(50,0 \%)$ & $13(52,0 \%)$ \\
Sonolência leve & $16(48.5 \%)$ & $11(45.8 \%)$ & $7(43,8 \%)$ & $11(42,3 \%)$ & $9(36,0 \%)$ \\
Sonolência moderada & $2(6,1 \%)$ & $3(12,5 \%)$ & $1(6,3 \%)$ & $1(3,8 \%)$ & $3(12,0 \%)$ \\
Sonolência severa & - & - & $1(6.3 \%)$ & $1(3.8 \%)$ & - \\
Total & $33(100 \%)$ & $24(100 \%)$ & $16(100 \%)$ & $26(100 \%)$ & $25(100 \%)$ \\
\hline
\end{tabular}

(-) Dados numéricos igual a zero. ABP - Aprendizagem Baseada em Problemas; TD - Metodologia Tradicional. 
Ao se comparar a sonolência entre os alunos estudados, foi possível observar que não houve resultado significante entre os períodos do curso $(\mathrm{p}=0,57)$, nem entre os alunos da metodologia ativa $(p=0,91)$ e tradicional $(\mathrm{p}=0,28)$, assim como entre as metodologias de aprendizagem $(\mathrm{p}=0,11)$, conforme mostra a tabela 4 .

$<<$ Inserir tabela 4

Tabela 4. Comparação da sonolência entre as metodologias de aprendizagem e os alunos do curso de Fisioterapia da Uepa, Belém-Pará, 2018

\begin{tabular}{|c|c|c|c|c|}
\hline Metodologia & Período & Média & p-valor & p-valor \\
\hline \multirow{3}{*}{ ABP } & $1^{\circ}$ ano & $11,27 \pm 3,24$ & & \multirow{5}{*}{ 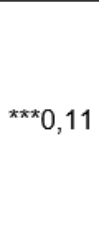 } \\
\hline & $2^{\circ}$ ano & $11,33 \pm 3,99$ & ${ }^{\star \star} 0,91$ & \\
\hline & $3^{\circ}$ ano & $11,75 \pm 4,87$ & \multirow{3}{*}{${ }^{\star \star} 0,28$} & \\
\hline \multirow{2}{*}{ Tradicional } & $4^{\circ}$ ano & $10,88 \pm 4,47$ & & \\
\hline & $5^{\circ}$ ano & $9,56 \pm 4,27$ & & \\
\hline p-valor & & ${ }^{\star} 0,57$ & & \\
\hline
\end{tabular}

*Análise de variância entre os anos do curso; **Análise de variância nas metodologias utilizadas; ***Análise de variância entre as metodologias utilizadas. Teste Anova (Um critério), $\mathrm{p} \leq 0,05$

Por fim, foi analisada, por meio da regressão linear múltipla, a correlação da percepção da QV e a sonolência nos discentes. Foi verificado que não houve correlação significante para os alunos do terceiro $(p=0,51)$, quarto $(p=0,38)$ e quinto ano $(p=0,31)$ do curso (tabela 5). Apesar de, em uma primeira análise (tabela 2), o quarto ano apresentar pior percepção da QV, esta não está relacionada diretamente com a sonolência.
No entanto, um dado interessante foi observado nos alunos do primeiro e segundo ano, ambos de forma significativa ( $p=0,04$ e $p=0,01$ respectivamente). Com relação aos alunos do primeiro ano, notou-se que aumenta 1,93 ponto na escala de sonolência quanto melhor a percepção de $\mathrm{QV}$ relacionada com o domínio relações sociais. Ou seja, quanto melhor a percepção da relação social, mais sonolenta a pessoa se percebe. Com relação ao domínio ambiente, foi possível notar que diminui 2,28 pontos na escala de sonolência quanto melhor a percepção de QV relacionada com o ambiente. Isto é, quanto melhor a percepção da $\mathrm{QV}$ relacionada com o ambiente, menos sonolenta a pessoa se sente, conforme mostra a tabela 5 . 
Tabela 5. Análise de regressão entre a sonolência (variável dependente) e a percepção da qualidade de vida (variável independente) em alunos do curso de Fisioterapia da Uepa, Belém-Pará, 2018

\begin{tabular}{|c|c|c|c|c|c|c|c|c|c|}
\hline & \multicolumn{3}{|c|}{$1^{\circ}$ ano } & \multicolumn{3}{|c|}{$2^{\circ}$ ano } & \multicolumn{3}{|c|}{$3^{\circ}$ ano } \\
\hline & $\mathrm{t}$ & $\mathrm{p}$ & $r$ & t & $\mathrm{p}$ & $r$ & $\mathrm{t}$ & $\mathrm{p}$ & $r$ \\
\hline \multicolumn{10}{|l|}{ WHOQOL-BREF } \\
\hline Físico & 0,0 & 0,92 & \multirow{5}{*}{$\begin{array}{l}F=2,61 \\
p=0,04^{*}\end{array}$} & $-1,5$ & 0,14 & & $-0,2$ & 0,84 & \multirow{5}{*}{$\begin{array}{l}F=0,91 \\
p=0,51\end{array}$} \\
\hline Psicológico & $-0,0$ & 0,99 & & 0,1 & 0,85 & & $-0,4$ & 0,67 & \\
\hline Relações sociais & 2,3 & $0,02^{*}$ & & $-3,4$ & $0,003^{*}$ & $\mathrm{p}=0,01^{*}$ & 0,7 & 0,48 & \\
\hline Ambiente & $-2,3$ & $0,02^{*}$ & & 1,6 & 0,10 & & 1,8 & 0,09 & \\
\hline \multirow[t]{3}{*}{ Qualidade de Vida } & $-0,4$ & 0,68 & & $-0,6$ & 0,50 & & $-0,6$ & 0,52 & \\
\hline & \multicolumn{5}{|c|}{$4^{\circ}$ ano } & \multicolumn{4}{|c|}{$5^{\circ}$ ano } \\
\hline & \multicolumn{2}{|r|}{$\mathrm{t}$} & \multicolumn{2}{|l|}{$p$} & $r$ & $\mathrm{t}$ & & $p$ & $r$ \\
\hline Físico & \multicolumn{2}{|c|}{$-1,4$} & \multicolumn{2}{|l|}{0,16} & & $-2,2$ & \multicolumn{2}{|c|}{0,03} & \\
\hline Psicológico & \multirow{2}{*}{\multicolumn{2}{|c|}{$\begin{array}{l}-0,6 \\
-0,8\end{array}$}} & \multicolumn{2}{|l|}{0,50} & $F=1,10$ & 0,0 & \multicolumn{2}{|c|}{0,92} & $F=1,28$ \\
\hline Relações sociais & & & 0,37 & & $p=0,38$ & 1,1 & \multicolumn{2}{|c|}{0,25} & $\mathrm{p}=0,31$ \\
\hline Ambiente & \multicolumn{2}{|r|}{$-0,5$} & \multicolumn{2}{|l|}{0,60} & & $-0,1$ & \multicolumn{2}{|c|}{0,85} & \\
\hline Qualidade de Vida & \multicolumn{2}{|r|}{1,6} & \multicolumn{2}{|l|}{0,10} & & 0,6 & \multicolumn{2}{|c|}{0,55} & \\
\hline
\end{tabular}

F (Regressão); T (Coeficiente de regressão). * Resultado estatisticamente significante. Teste de Regressão Linear Múltipla $(\mathrm{p} \leq 0,05)$.

Para os alunos do segundo ano, observou-se que diminui 3,65 pontos na escala de sonolência quanto melhor a percepção de QV relacionada com relações sociais. Ou seja, quanto melhor a percepção na relação social, menos sonolenta a pessoa se percebe (tabela 5). 


\section{DISCUSSÃO}

O curso de Fisioterapia da Uepa, desde o ano de 2016, implantou um novo projeto pedagógico migrando de um modelo de aprendizagem tradicional para o de metodologia ativa. No entanto, sabe-se que as metodologias ativas requerem um esforço maior do estudante uma vez que este será responsável pela sua aprendizagem. Porém, também se sabe que, na TD, existe a imposição de conteúdos excessivos e que esses alunos precisam assimilá-los até a culminância da avaliação da aprendizagem. Sabe-se que a saúde mental dos alunos é essencial para a vida acadêmica e para o futuro profissional. Pensando nisso, os autores se questionaram: há alterações na percepção da QV e sonolência dos alunos entre os diferentes períodos do curso e entre as metodologias utilizadas? Essa era uma pergunta intrigante e que se precisava conhecer para que se pudesse fazer algo dentro da própria universidade e do curso de Fisioterapia.

Sabe-se que, atualmente, a cultura da aprendizagem se torna mais natural, dinâmica e prepara o aluno para o mundo, diferindo da forma tradicional, focada em apenas transmitir conhecimento, com alunos inertes e professores conteudistas e competitivos. Essa mudança na aprendizagem gera novas demandas impostas aos alunos, que, por vezes, não estão preparados para lidar com essa situação; e os conflitos de ordem pessoal, familiar e social acabam tomando proporções muito maiores $^{10,11}$.

Em relação à análise isolada da QV nos alunos do curso de Fisioterapia, foi possível notar que apenas os alunos do quarto ano apresentaram pior percepção em relação ao domínio físico e ambiente; o que diverge do encontrado no estudo de Alves e colaboradores ${ }^{12}$ em que verificaram que, durante o curso médico, a $\mathrm{QV}$ sofre desgastes no domínio psicológico. Já em outros estudos ${ }^{13,14}$, com estudantes de Fisioterapia, a pior percepção da QV foi no domínio ambiente. Este estudo, em parte, corrobora esses autores, uma vez que mostra alteração no domínio ambiente.

Vários universitários, ao ingressar na universidade, mudam de cidade, o que leva a um distanciamento de amigos e familiares, repercutindo nos domínios sociais e ambientais desses alunos ${ }^{15}$. Neste estudo, não foi observado esse padrão nos alunos que realizam as primeiras séries do curso, o que seria natural de se observar. Houve esse padrão nos alunos do quarto ano que estão experimentando outro momento de transição acadêmica, que é a fase de pré-ingresso no estágio obrigatório.

$\mathrm{Na}$ universidade, os estudantes são encorajados a serem independentes em sua formação, buscando conteúdos e ensinamentos em atividades curriculares e extracurriculares ${ }^{16}$. Por essa razão, seria esperado observar resultados significantes para os alunos do primeiro ano do curso, uma vez que ainda não estão familiarizados com o ensino superior e, muito menos, com a metodologia ativa na qual está inserido. Porém, neste estudo, observou-se essa situação nos discentes do quarto ano - período que ainda faz parte do modelo tradicional e no qual há uma imensa exposição dos alunos a diversas áreas da Fisioterapia com inúmeros conteúdos a serem estudados. Talvez isso explique os resultados obtido neste estudo.

Essa grande carga de atividades curriculares e, também, extracurriculares para os discentes do quarto ano pode ser uma explicação da pior percepção no domínio físico encontrado neste estudo. Muitos alunos podem se sentir amedrontados em virtude de se aproximar o ano do estágio obrigatório, fazendo com que eles estudem mais e durmam menos, levando a um desgaste físico - assim como afirma outro estudo ${ }^{17}$, que atribui a baixa percepção da QV para o domínio físico a múltiplos fatores que acabam comprometendo a saúde física, como a carga horária extenuante, a multiplicidade de atividades, a falta de organização do tempo, a irregularidade do sono, a ansiedade, a alimentação inadequada e o sedentarismo.

A sobrecarga dessas atividades pode influenciar no sono dos alunos e, consequentemente, no seu rendimento escolar. Este estudo aponta que os alunos do segundo ano foram os que apresentaram índices maiores de sonolência $(58,3 \%)$, seguido do terceiro ano $(56,4 \%)$ e do primeiro ano (54,6\%); e os anos seguintes estiveram abaixo de $50 \%$. Isso corrobora o estudo de Martini e colaboradores6, o 
qual evidenciou que $51,75 \%$ dos estudantes do curso de Fisioterapia apresentaram uma qualidade do sono ruim.

Almeida e colaboradores ${ }^{7}$, em seu estudo, mostraram que, em estudantes de Fisioterapia, 28,46\% apresentavam sonolência diurna; o que diverge deste estudo que encontrou altos índices de sonolência diurna, principalmente naqueles estudantes pertencentes aos períodos iniciais do curso.

Alguns estudos relatam que, nos finais de semana, os estudantes dormem um pouco mais ${ }^{18}$, supondo que essas horas de sono foram perdidas durante a semana, tentando recuperá-las. Assim, estar um pouco mais na cama aos finais de semana pode apresentar importante papel na compensação do sono ${ }^{19}$.

Alterações no sono podem impactar negativamente em várias funções do estudante, levando a maior sonolência diurna, proporcionando diminuição no desempenho escolar como mostrado por Cardoso e colaboradores ${ }^{9}$, os quais encontraram uma correlação positiva entre a privação do sono e a diminuição do desempenho acadêmico e profissional entre estudantes de medicina.

A Sonolência Diurna Excessiva (SDE) é um distúrbio que interfere tanto na QV quanto no desempenho cognitivo, atencional, profissional e no relacionamento social $^{20-22}$. Neste estudo, buscou-se a relação da QV com a sonolência, em que foi possível observar resultados significantes apenas nos alunos pertencentes ao primeiro e segundo ano. Nos alunos do primeiro ano, percebeu-se que quanto melhor a percepção da QV no domínio relação social, maior era a sonolência diurna; e quanto melhor a percepção da QV no domínio ambiente, menor era a sonolência diurna. Já para os alunos do segundo ano, foi o inverso: quanto melhor a percepção da QV no domínio relação social, menor era a sonolência diurna. Nota-se que o fator relação social, em ambos os anos, está afetando a sonolência de forma diferente.

Soares e Pettre ${ }^{23}$ afirmam que, no processo de adaptação ao ensino superior, as relações sociais entre os estudantes são diversas, como idades diferentes, de experiência de vida, de características pessoais e dos papéis sociais. Essa diversidade, para que não gere conflitos, exige habilidade do estudante nas relações interpessoais/ sociais. Talvez isso aconteça com os alunos que estão avançando no curso, podendo ser mais observada essa relação com alunos de séries mais avançadas - bem como o fator ambiental, pois o curso de Fisioterapia apresenta uma estrutura que surpreende positivamente os alunos que vêm do ensino médio com uma visão deturpada de universidade pública.

Além disso, o entusiasmo para iniciar o curso superior e se familiarizar sobre a futura profissão também reflete nos aspectos psicoemocionais dos estudantes, criando expectativas no que diz respeito à relação aluno-professor, esperando que eles possam repassar mais do que ensinamentos, como também a paixão pela sua profissão ${ }^{24}$. Bardagi ${ }^{25}$ mostra a importância de analisar os estudantes em diferentes anos do mesmo curso, uma vez que se espera, com o passar do tempo, um amadurecimento da sua vida acadêmica. Neste estudo, foi realizado o que o autor relatou, e não somente no mesmo curso, mas também se avaliou como esse aluno se comporta em metodologias diferentes.

Ressalta-se que, embora se tenha obtido resultados interessantes, é de suma importância que haja mais pesquisas que avaliem cuidadosamente essas variáveis estudadas. Aponta-se, ainda, que há limitações no que se refere à utilização de escalas subjetivas em busca de informações que são cruciais no acompanhamento mental desses alunos. No entanto, são estratégias que podem ser utilizadas como rastreamento dessas alterações e realizar o devido encaminhamento para profissional capacitado. A coordenação do curso também pode avaliar suas programações, procurando melhorá-las e adequá-las às necessidades dos alunos, enfatizando $\mathrm{a}$ potencialização das habilidades de aprendizagem, sem a interferência de problemas com o sono que altera a QV do aluno.

\section{CONCLUSÕES}

Os resultados encontrados demonstram que, com relação à $\mathrm{QV}$, apenas o quarto ano apresentou menor percepção 
para o domínio físico e ambiente, e quando comparado aos demais anos, apenas o domínio físico foi menor. Para a sonolência, mesmo sem significância estatística, houve altos índices para o segundo ano do curso e entre as metodologias ativas. Por fim, observou-se correlação entre QV e sonolência diurna apenas nos alunos de metodologia ativa e aqueles pertencentes ao primeiro e segundo ano. No primeiro ano, foi observado que quanto melhor percepção da QV no domínio relação social, mais sonolento se percebe. Outrossim, para o domínio ambiente, quanto melhor a percepção, menos sonolento. Já para o segundo ano, quanto melhor percepção da QV no domínio relação social, menos sonolento se percebe.

\section{REFERÊNCIAS}

1. Costa PHV, Silva FS, Machado CJ. Nível de atividade física e qualidade de vida dos estudantes de fisioterapia de uma instituição privada de ensino superior. Rev Interdiscip Ciênc Méd. 2018;1(2):46-53.

2. Silva AL, Smaidi K, Pires MHR, Pires OC. Prevalência da dor crônica e fatores associados em estudantes de medicina. Rev Dor. 2017;18(2):108-111.

3. Carvalho MCP. Levantamento da situação de saúde mental e uso de ansiolíticos e antidepressivos por acadêmicos do curso de odontologia de uma universidade do sul de minas Gerais. Rev. Univ. Vale Rio Verde. 2017;15(1):489-496.

4. Nogueira MJ, Sequeira C. A saúde mental em estudantes do ensino superior. Relação com o género, nível socioeconómico e os comportamentos de saúde. Rev Port Enferm Saúde Mental. 2017;5:51-56.
5. Moutinho ILD, Maddalena NCP, Roland RK, Lucchetti ALG, Tibiriçá SHC, Ezequiel OS, et al. Depression, stress and anxiety in medical students: A cross--sectional comparison between students from different semesters. Rev Assoc Med Bras. 2017;63(1):21-28

6. Martini M, Brandalize M, Louzada FM, Pereira EF, Brandalize D. Fatores associados à qualidade do sono em estudantes de Fisioterapia. Fisioter Pesq. 2012;19(3):261-267.

7. Almeida JOS, Siqueira PPS, Lima AMJ, Brasileiro-Santos MS, Galindo Filho VC. Sonolência diurna e qualidade do sono em estudantes universitários de fisioterapia. ConScientiae Saúde. 2011;10(4):201-209.

8. Fleck MP, Louzada S, Xavier M, Chachamovich E, Vieira G, Santos L, et al. Application of the Portuguese version of the abbreviated instrument of quality life WHOQOL-bref. Rev Saude Publica. 2000;34(2):178-83.

9. Cardoso HC, Bueno FCC, Mata JC, Alves APR, Jochims I, Vaz Filho IHR, et al. Avaliação da qualidade do sono em estudantes de Medicina. Rev Bras Educ Med. 2009;33(3):349-355.

10. Lopes RC, Castro DT. A importância das tecnologias digitais no processo de ensino e aprendizagem. Rev Humanidades Inovação. 2015;2(2):75-82.

11. Marques MCPD, Gomes JPBA, Gomes AJA. A integração das tecnologias digitais de informação e comunicação (TDIC) no ambiente escolar. Rev Ágora. 2017;1(1):1-25.

12. Alves JGB, Tenório M, Anjos AG, Figueroa JN. Qualidade de vida em estudantes de Medicina no início e final do curso: avaliação pelo Whoqol-bref. Rev Bras Educ Med. 2010;34(1):91-6. 
13. Alfredo PP, Biondi JCL, Manna V. Avaliação da qualidade de vida e estresse em acadêmicos do curso de Fisioterapia. J Health Sci Inst. 2016;34(4):224-30.

14. Goncalves MS, Santos LFS, Pereira SAP, Rodrigues CC, Serafim GML. Análise da qualidade de vida dos discentes do curso de graduação em Fisioterapia da Universidade Estadual do Piauí, através do WHOQOL-BREF. Fisioterapia Brasil. 2014;15(1):10-15.

15. Teixeira MAP, Dias ACG, Wottrich SH, Oliveira AM. Adaptação à universidade em jovens calouros. Psicol Esc Educ. 2008;12(1):185-202.

16. Santos AAA, Mognon JF, Lima TH, Cunha NB. A relação entre vida acadêmica e a motivação para aprender em universitários. Psicol Esc Educ. 2011;15(2):283-290.

17. Leite ACB, Grillo LP, Caleffi F, Mariath AB, Stuker H. Qualidade de vida e condições de saúde de acadêmicos de Nutrição. Espac Saude. 2011;13(1):82-90.

18. Brandalize M, Pereira EF, Leite N, Filho GL, Louzada FM. Effect of morning school schedule on sleep and anthropometric variables in adolescents: A follow-up study. Chronobiol Int. 2011;28(9): 779-85.

19. Wing YK, Li SX, Li AM, Zhang J, Shan AP. The effect of weekend and holiday sleep compensation on childhood overweight and obesity. Pediatrics. 2009;124(5):994-1000.

20. Connor J, Norton R, Ameratunga S, Robinson E, Civil I, Dunn R, et al. Driver sleepiness and risk of serious injury to car occupants: population based case control study. BMJ. 2002;324(7346):1125.
21. Canani SF, Barreto SSM. Sonolência e acidentes automobilísticos. J Pneumologia. 2001;27(2):94-6.

22. Mulgrew AT, Ryan CF, Fleetham JA, Cheema R, Fox N, Koehoorn M, et al. The impact of obstructive sleep apnea and daytime sleepiness on work limitation. Sleep Med. 2007;9(1):42-53.

23. Soares AB, Pettre ZAP. Habilidades sociais e adaptação à universidade: Convergências e divergências dos construtos. Anál Psicol. 2015;2(33):139-151.

24. Castro AKSS, Teixeira MAP. A evasão em um curso de psicologia: uma análise qualitativa. Psicol Estud. 2013;18(2):199-209.

25. Bardagi MP. Evasão e comportamento vocacional de universitários: estudos sobre o desenvolvimento de carreira na graduação [tese]. Porto Alegre (RS): Universidade federal do Rio Grande do Sul; 2007. 